\section{Gabriella Buzzi}

M.G. Buzzi (西)

IRCCS Neuromed

Via Atinense 18, I-86077 Pozzilli (IS), Italy

e-mail: buzzi@neuromed.it

Tel.: +39-0865-929304

Fax: +39-0865-925351

\section{Triptans in clinical practice: the basic scientist's point of view}

\begin{abstract}
Since the observation that sumatriptan, the first triptan to be synthetized as a specific antimigraine compound, was able to block neurogenic inflammation (NI) in intra- and extracranial rat tissues, the experimental model for NI development has been used to predict the efficacy in aborting migraine pain of other compounds with activity on 5-HT1B/1D receptors. Most of the drugs active on 5-HT1 receptors that have been tried in the NI model effectively inhibited or decreased this response, although some of them lack of clinical efficacy on migraine pain, suggesting that pain relief does not correlate with NI blockade. A central pathophysiological mechanism has been advocated since migraine attack is considered to be a discharge from a central "generator", probably located in the brainstem.
\end{abstract}

The hypothesis is supported by experimental data showing that triptans inhibit excitability of neurons in the trigeminal nucleus caudalis when locally applied. This experimental model does not rule out the involvement of peripheral blockade during migraine attack. Probably, neurophysiological studies in humans provide better information than do experimental animal models about central mechanisms of action of triptans. Receptor pharmacology is also important for understanding beneficial or undesidered side effects other than pain relief. Biochemical studies are therefore needed to better understand how triptans work and to design new abortive or preventive drugs.

Key words Triptans $\cdot$ Neurovascular system $\cdot$ Serotonin receptors $\cdot \mathrm{CNS}$

\section{Introduction}

Ten-year experience with sumatriptan and, to a lesser extent, with second-generation triptans, is probably enough to make up our minds about the revolutionary therapeutical tools they are in clinical practice. However, thinking from other points of view may offer clues for better prescribing one triptan rather than others and for choosing among them according to patients' needs. Therefore, it is useful to rethink triptans as a class with several mechanisms beyond the pure clinical efficacy. These mechanisms are reviewed and future directions for clinical research studies for further understanding and drug development are discussed.

\section{The relevance of neurovascular mechanisms}

Since the observation that sumatriptan, the first triptan to be synthetized as a specific anti-migraine compound, was able to block neurogenic inflammation (NI) in intra- and 
extracranial rat tissues [1], the experimental model for NI development has been used in the last decade to predict efficacy in aborting migraine pain of other compounds with activity on 5-HT1B/1D receptors. Briefly, NI represents vasodilatation and plasma protein extravasation that follows release of vasoactive peptides such as CGRP, SP and NKA from nerve endings upon activation of sensory fibers. As an animal model of migraine, NI is induced in rat dura mater and other trigeminally innervated cephalic tissues, by either systemic administration of capsaicin (the pungent ingredient of hot pepper which is able to depolarize sensory fibers, or to abolish their functions when given to neonate animals) or by stimulating trigeminal sensory fibers by electrical stimuli [2]. Systemic administration of substance $\mathrm{P}$ is also able to induce tissue edema without direct involvement of sensory fibers [1].

Sumatriptan effectively inhibited single components of the neurogenic response to peripheral trigeminal ganglion stimulation, such as CGRP increase in plasma from the superior sagittal sinus (SSS) [2] and mast cell activation and degranulation in the tissues [3]. Sumatriptan is also effective in reducing the increase of CGRP in the jugular vein during migraine attacks [4]. However, this observation is not clinically relevant since plasma CGRP levels increase at the beginning of electrical stimulation of the trigeminal ganglion but decrease when the stimulation is still running [5]. The decrease in peptide level during a spontaneous migraine attack may, therefore, be spontaneous as well, and not related to drug activity. Administration of sumatriptan after the onset of an attack may, instead, block further release of the peptide or not, thus explaining effectiveness or recurrence as seen in certain patients receiving triptans and experiencing headache recurrence within 24 hours.

In this view, recurrence rate (RR) may depend on a drug's half-life. In fact sumatriptan, zolmitriptan, rizatriptan and almotriptan have the highest RR (30\%-40\%) and the shortest half-life $(2.0-3.5 \mathrm{~h})$, eletriptan and naratriptan have a lesser RR (20\%-30\%) and a longer half-life (5-6 h), while frovatriptan has the longest half-life $(25 \mathrm{~h})$ with the lowest RR (11\%) [6].

Most of the drugs active on 5-HT1 receptors that have been tried in the NI model effectively inhibited or decreased this response, although some of them lack of clinical efficacy on migraine pain. This may reflect the need for dose adjustment, or indicate that the efficacy on experimental NI does not need recruitment of vascular receptors as "coworkers" in the inhibition of edema, whereas the same may be necessary in clinical practice. This may be the case for the conformationally restricted sumatriptan analog CP122,288 a compound that shows high potency in NI [7] but not on pain [8] and is devoid of 5-HT1B-mediated vascular action.
The role of the 5-HT1F receptor is indicated by the observation that selective agonists (such as LY344864) attenuate c-fos expression in the trigeminal nucleus caudalis (TNC) following intracisternal capsaicin (another model to activate trigeminal sensory fibers) [9].

An interesting observation that makes the neurogenically mediated activity quite relevant to triptan mechanism of action is that patients with migraine attacks associated with neurovascular signs on the side of pain (e.g. lacrimation, conjunctival injection, rhinorrea, tissue edema) show a higher degree of clinical response to triptans as compared to the general migraine population [10]. These data provide a clue for identifying triptan-responders among migraineous patients and emphasize the need for strict clinical trials regarding the use of triptans in non-migraineous head pains, namely the trigeminal autonomic cephalalgias (TACs) [11].

\section{Other drug-related CNS components}

Given the central origin of migraine attacks, a central site of action, due to penetration in the CNS, has been provided as an additional mechanism of second-generation triptans, and several compounds are now on the market or at an advanced pre-clinical stage (key sites of triptan action are reviewed in [12]). The central mechanism has been advocated since the migraine attack is considered to be a discharge from a central "generator", probably located in the brainstem [13]. This belief is supported by experimental data showing that triptans are able to inhibit excitability of neurons in the TNC when locally applied [14]. However, when the same neurons are excited following peripheral trigeminal fiber activation, their activity may be inhibited if the peripheral component is blocked by systemic administration of sumatriptan, a drug that does not cross the blood-brain barrier (BBB) at therapeutical doses. Therefore, the model lacks clinical relevance and does not clarify whether the trigeminovascular system as the site of triptan action is needed or not.

Neurophysiological studies in humans provide better information than experimental animal models about the central mechanism of action of triptans. Particularly, IDAP (intensity dependence of the cortical auditory evoked potentials) increased after zolmitriptan administration in migraine patients and normal subjects [15]. High IDAP variability is reported in normal subjects receiving naratriptan and zolmitriptan [16], thus explaining the high variability of triptans in terms of clinical efficacy in migraine attacks and minimizing the relevance of a central action for triptans. 5HT1B/D agonists inhibit NMDA receptor-evoked NO synthase and cGMP concentrations in brain cortex slices [17], as seen with 5-HT via 5-HT1A receptors [18]. Obviously, this mechanism requires in vivo penetration in the CNS. The 
use of anti-epileptic drugs as migraine prophylaxis requires further studies concerning interference with CNS-penetrating triptans.

When considering the clinical efficacy of triptans, the central action does not seem to have added any further advance on pain and RR. In fact, the degree of pain relief and pain freedom, as well as RR, is about the same for sumatriptan and the newly synthetized triptans and it seems to depend upon drug half-life rather than on other mechanisms. The gain obtained with the newer drugs concerns the lower incidence of peripheral (probably vascular) side effects. However, central side effects are increased [19].

Although rarely considered, other serotonin receptors are involved when triptans are given: 5-HT1F receptor is activated by eletriptan, 5-HT1A by zolmitriptan, 5-HT7 by eletriptan and zolmitriptan, and 5-HT2B by zolmitriptan. Each of these receptors mediates effects other than pain relief. Anxiety, that usually accompanies the attack and is possibly increased by activation of 5-HT1B/1D [20], is relieved by activating 5-HT1A receptor but the same can worsen gastrointestinal discomfort. Coronary vasodilation is mediated by 5 -HT7 receptor and its activity should be advocated when using 5-HT1B/1D receptor agonists. Therefore, a central action might be undesired and should be better evaluated not only in terms of clinical response and side effects. Based on pharmacological profile, the best drug should be designed as having $5-\mathrm{HT} 1 \mathrm{~B} / 1 \mathrm{D}$ and $1 \mathrm{~F}$ potency as well as lack of activity on 5-HT2B/C receptors mediating vasodilatation in the cephalic circulation. In fact, their activation is responsible for NO synthesis through mobilization of intracellular calcium ions and activation of NOS. NO acts as a vasodilator and facilitates release of vasoactive peptides from trigeminal nerve endings. These functions are probably responsible for $\mathrm{mCPP}$-induced headache.

All 5-HT receptors (excluding 5-HT3) belong to the first class of the G-protein coupled receptor superfamily. 5-HT1 receptor subtypes are coupled to a Gi protein. Their effects include inhibition of adenyl cyclase activity and inhibition of voltage-sensitive $\mathrm{Ca}^{2+}$ channels. Involvement of $\mathrm{Gi}$ proteins in the modulation of pain perception has been established and lack of its function enhances the sensitivity to pain. A hypofunctionality of Gi proteins has been recently documented in migraine patients [21]. Restoration of normal function of this system may be a possible explanatory key for the efficacy of short-term prophylaxis with triptans, for example in menstrual migraine.

\section{References}

1. Buzzi MG, Moskowitz MA (1990) The antimigraine drug, sumatriptan (GR 43175), specifically blocks plasma extravasation from blood vessels in dura mater. Br J Pharmacol 99:202-206

2. Markowitz S, Saito K, Moskowitz MA (1987) Neurogenically mediated leakage of plasma protein occurs from blood vessels in dura mater but not brain. J Neurosci 7:4129-4136

3. Buzzi MG, Dimitriadou V, Theoharides TC, Moskowitz MA (1992). 5-Hydroxytryptamine receptor agonists for the abortive treatment of vascular headaches block mast cell, endothelial and platelet activation within the rat dura mater after trigeminal stimulation. Brain Res 583:137-149

4. Goadsby PJ, Edvinsson L (1993). The trigeminovascular system and migraine: studies characterizing cerebrovascular and neuropeptide changes seen in humans and cats. Ann Neurol 33:48-56
5. Buzzi MG, Carter WB, Shimizu T, Heath H III, Moskowitz MA (1991) Dihydroergotamine and sumatriptan attenuate levels of CGRP in plasma in rat superior sagittal sinus during electrical stimulation of the trigeminal ganglion. Neuropharmacology 30:1193-1200

6. Tfelt-Hansen P, Devries P, Saxen PR (2000) Triptans in migraine: a comparative review of pharmacology, pharmacokinetics and efficacy. Drugs 60:1259-1287

7. Lee WS, Moskowitz MA (1993) Conformationally restricted sumatriptan analogues, CP-122,288 and CP122,638 exhibit enhanced potency against neurogenic inflammation in dura mater. Brain Res 626:303-305

8. Roon KI, Olesen J, Diener HC, Ellis P, Hettiarachchi J, Poole PH, Christianssen I, Kleinermans D, Kok JG, Ferrari MD (2000) No acute antimigraine efficacy of CP-122,288, a highly potent inhibitor of neurogenic inflammation: results of two randomized, double-blind, placebo-controlled clinical trials. Ann Neurol 47:238-241
9. Mitsikostas DD, Sanchez del Rio M, Moskowitz MA, Waeber C (1999) Both 5-HT1B and 5-HTT1F receptors modulate c-fos expression within rat trigeminal nucleus caudalis. Eur J Pharmacol 26:271-277

10. Barbanti P, Buzzi MG, Fabbrini G, Vanacore M, Pesare G, Lenzi GL, Cerbo R (2000) Neurovascular symptoms during migraine attacks: response to triptans. Cephalalgia 20:428-429

11. Goadsby PJ, Lipton RB (1997) A review of paroxysmal hemicrania, SUNCT syndrome and other short-lasting headaches with autonomic feature, including new cases. Brain 120:193-209

12. Hargreaves RJ (2000) Pharmacology and potential mechanisms of action of rizatriptan. Cephalalgia 20[Suppl 1]:2-9

13. Weiller C, May A, Limmroth V, Juptner M, Kaube H, Schayck RV, Coenen HH, Diener HC (1995) Brain stem activation in spontaneous human migraine attacks. Nat Med 1:658-660 
14. Storer RJ, Goadsby PJ (1997) Microiontophoretic application of serotonin $(5 \mathrm{HT}) 1 \mathrm{~B} / 1 \mathrm{D}$ agonists inhibits trigeminal cell firing in the cat. Brain 120:2171-2177

15. Proietti-Cecchini A, Afra J, Schoenen J (1997) Intensity dependence of the cortical auditory evoked potentials as a surrogate marker of central nervous system serotonin transmission in man: demonstration of central effect for the 5HT1B/1D agonist zolmitriptan (311C90, Zomig). Cephalalgia 17:849-854

16. Roon KJ, Sandor PS, Schoonman GG, Lamers FPL, Schoenen J, Ferrari MD, van Dijk JG (1999) Auditory evoked potential in the assessment of central nervous system effects of antimigraine drugs. Cephalalgia 19:880-885
17. Stepien A, Chalimoniuk M, Strosznajder J (1999) Serotonin 5HT1B/1D receptor agonists abolish NMDA receptor-evoked enhancement of nitric oxide synthase activity and cGMP cencentration in brain cortex slices. Cephalalgia 19:859-865

18. Strosznajder J, Chalimoniuk M, Samochocki M (1996) Activation of serotoninergic 5HT1A receptor reduces $\mathrm{Ca} 2+$ and glutamatergic receptorevoked arachidonic acid and NO/cGMP release in adult hippocampus. Neurochem Int 28:439-444

19. Buzzi MG, Fabbri L (2000) New methods for evaluating acute treatments in migraine. Funct Neurol

3[Suppl]:192-195
20. Zhuang X, Gross C, Santarelli L, Compan V, Trillat AC, Hen R (1999) Altered emotional states in knockout mice lacking 5-HT1A or 5-HT1B receptors. Neuropsychopharmacology 21:52S-60S

21. Galeotti N, Ghelardini C, Zoppi M, Del Bene E, Raimondi L, Beneforti E, Bartolini A (2001) Hypofunctionality of $\mathrm{Gi}$ proteins as aetiopathogenetic mechanism for migraine and cluster headache. Cephalalgia 21:38-45 